Ambiente \& Água - An Interdisciplinary Journal of Applied Science
ISSN 1980-993X - doi:10.4136/1980-993X
www.ambi-agua.net
E-mail: ambi.agua@gmail.com

\title{
Redundância entre métricas da qualidade ambiental de riachos em paisagem agrícola
}

\author{
doi:10.4136/ambi-agua.1665
}

Received: 20 May 2015; Accepted: 17 Sep. 2015

\author{
Marcel Okamoto Tanaka*; Andréa Lúcia Teixeira de Souza; \\ Alexandre Kannebley de Oliveira; Luiz Eduardo Moschini \\ Universidade Federal de São Carlos (UFSCar), São Carlos, SP, Brasil \\ Departamento de Ciências Ambientais \\ *Autor correspondente: e-mail: marcel@ufscar.br, \\ altdesouza@gmail.com,pako@ufscar.br, lemoschini@ufscar.br
}

\section{RESUMO}

Indicadores ambientais são amplamente usados para avaliação da qualidade dos corpos d'água. Como o monitoramento envolve alto custo de amostragem, e a construção de índices multimétricos pressupõe métricas não correlacionadas, este estudo avaliou se havia correlação entre métricas que caracterizam o uso e cobertura do solo, composição da zona ripária e estrutura da floresta, bem como correlações entre indicadores das comunidades de macroinvertebrados e peixes. O estudo foi feito em 12 afluentes de baixa ordem dos rios Jacaré-Pepira e Jacaré-Guaçu, formando um gradiente de estresse ambiental no estado de São Paulo. Não houve correlação entre as métricas relacionadas ao uso e cobertura do solo, e apenas a proporção de pastos foi negativamente correlacionada com algumas métricas da estrutura da floresta ripária. Assim, tanto as informações do uso e cobertura do solo quanto sobre a composição da zona ripária e estrutura das florestas podem ser importantes na avaliação ambiental de riachos. As métricas relacionadas às comunidades de macroinvertebrados foram correlacionadas entre si, assim como a maioria das métricas relacionadas às comunidades de peixes. Estudos propondo índices multimétricos não encontraram correlação entre estas variáveis, mas avaliaram riachos em situações extremas (preservados vs. impactados), sendo os riachos impactados influenciados por diferentes condutores de degradação, o que pode resultar em respostas distintas das comunidades biológicas. Os resultados deste estudo sugerem que o desenvolvimento de índices deve ser bastante específico para cada região estudada, e que muitas vezes as avaliações são feitas em relação a condutores de impactos específicos, que podem variar entre diferentes sistemas.

Palavras-chave: biomonitoramento, estrutura de florestas ripárias, macroinvertebrados, peixes, uso e cobertura do solo.

\section{Redundancy among environmental quality metrics of streams in an agricultural landscape}

\begin{abstract}
Biological indicators are widely used for the evaluation of the environmental quality of waterbodies. Since monitoring involves high sampling costs, and the creation of multimetric
\end{abstract}


indices assumes uncorrelated metrics, this study evaluated whether there was correlation among metrics that characterize land use and cover, riparian zone composition and forest structure, as well as correlations among indicators of macroinvertebrate and fish communities. The study was conducted on 12 low-order tributaries of the Jacaré-Pepira and Jacaré-Guaçu Rivers, forming a gradient of environmental stress in the central region of São Paulo State. There were no correlations among land use metrics, and only pasture cover was correlated with some metrics of riparian forest structure. Therefore, both information on land use and riparian composition and forest structure can be important in the environmental assessment of streams. All metrics related to macroinvertebrate communities were correlated to each other, as well as most metrics related to fish communities, although most studies proposing multimetric indices have not found correlation among these metrics. Those studies evaluated streams in extreme situations (preserved vs. impacted), and impacted streams were influenced by different degradation drivers, which may result in distinct responses by the biological communities. The results of the present study suggest that the development of indices must be specific to the studied region, and that frequently the evaluations are carried out in relation to specific drivers of impact, which may vary for different systems.

Keywords: biomonitoring, fish, land use, macroinvertebrates, riparian forest structure.

\section{INTRODUÇÃO}

A avaliação da qualidade ambiental é um procedimento básico na gestão de corpos d'água doce e bacias hidrográficas (Ritter e Shirmohammadi, 2010). A construção de indicadores multimétricos para o monitoramento dos corpos d'água é complexa, e muitas vezes envolve o uso de métricas diversas para sua aplicação, podendo envolver variáveis físicas, químicas e biológicas. Análises físico-químicas da água são comumente empregadas, mas refletem as características momentâneas dos corpos d'água, com respostas rápidas a impactos pontuais; por outro lado, o uso de métricas biológicas propicia a detecção de impactos crônicos, não pontuais, com respostas cumulativas das populações e comunidades biológicas a alterações ambientais (Friberg et al., 2011). Duas categorias principais de indicadores têm sido utilizadas para se avaliar e prever a qualidade ambiental dos corpos de água doce: 1) métricas relacionadas ao uso e cobertura do solo e características da zona ripária, e 2) métricas das comunidades biológicas aquáticas (Barbour et al., 1999; Goforth e Bain, 2004; Clapcott et al., 2012).

Nas escalas da paisagem e da zona ripária, diversos índices multimétricos e protocolos de análise foram propostos para avaliar a qualidade da água de riachos, geralmente relacionada às alterações antrópicas do uso e cobertura do solo e à degradação das zonas ripárias. Por exemplo, Ometo et al. (2000) propuseram o índice LUI (Land Use Index) a partir de diferentes pesos dados a nove métricas de usos do solo, encontrando relações significativas entre os valores do índice e variáveis da qualidade da água de riachos no Estado de São Paulo. Posteriormente, Goforth e Bain (2010) propuseram o índice WHEBIP (Watershed Habitat Evaluation and Biotic Integrity Protocol) a partir de 12 métricas descrevendo o uso e cobertura do solo e características da zona ripária, obtidas a partir da análise de fotos aéreas (1:24.000), e encontraram relações significativas entre este índice e a qualidade biológica de riachos nos EUA. Outros estudos avaliando a relação entre o uso e cobertura do solo e a qualidade da água incluem os trabalhos de Miserendino et al. (2011) que encontraram relação entre a intensidade de uso do solo e a qualidade da água de riachos na Patagônia, e Clapcott et al. (2012) que quantificaram a relação entre categorias básicas de cobertura do solo e indicadores de integridade funcional de riachos usando uma base de dados nacional dos EUA. 
Recentemente, Sheldon et al. (2012) mostraram que o uso e cobertura do solo, em especial a cobertura da floresta ripária adjacente aos cursos d'água, era fortemente correlacionada com a integridade dos ecossistemas lóticos. De fato, diversos estudos mostram forte influência da composição da zona ripária e estrutura das florestas ripárias na qualidade da água de riachos (Merritt et al., 2010; Souza et al., 2013; Fernandes et al., 2014), sendo que a largura das florestas ripárias é um dos principais referenciais para políticas de preservação de corpos d'água em todo o mundo.

Por outro lado, indicadores biológicos das comunidades aquáticas têm sido usados na avaliação da qualidade da água há mais tempo. Por exemplo, comunidades de macroinvertebrados são consideradas bons indicadores por incluírem alta diversidade de espécies, com diferentes grupos que respondem a tipos distintos de perturbações, terem ciclos de vida suficientemente longos, serem relativamente sedentários para representar as condições locais e ocorrerem na maioria dos habitats aquáticos em grande abundância, sendo sua coleta de baixo custo (Friberg et al., 2011). Estas características possibilitam sua aplicação em diversos sistemas e com diversas abordagens de avaliação, incluindo o uso de índices multimétricos (Buss et al., 2015). Peixes constituem outro grupo bastante usado em estudos de biomonitoramento, por responderem a alterações da qualidade da água e degradação do habitat. Por exemplo, Angermeier e Karr (1986) propuseram um índice multimétrico de integridade biológica (IBI) de riachos, considerando aspectos da diversidade e estrutura funcional das comunidades de peixes. Estes trabalhos influenciaram o desenvolvimento de diversos outros índices de integridade biológica em diferentes regiões do mundo, baseados nas características da ictiofauna regional, conforme proposto em Barbour et al. (1999). Exemplos no sudeste brasileiro incluem a adaptação do IBI para riachos da bacia do Alto Rio Paraná (Casatti et al., 2009).

A obtenção de dados para o monitoramento biológico, entretanto, envolve vários custos, desde a amostragem e procedimentos em laboratório, até a análise e curadoria de dados (Caughlan e Oakley, 2001, McDonald-Madden et al., 2010). Desta forma, métricas correlacionadas ou não informativas aumentam o custo da amostragem, demandando recursos que poderiam ser usados em outras etapas do monitoramento (Braun e Reynolds, 2012). Além disso, a construção de índices multimétricos de qualidade ambiental a partir de métricas de uso e cobertura do solo, características da zona ripária e das comunidades biológicas envolve várias etapas. De acordo com Hering et al. (2006), uma das etapas importantes é a seleção das métricas a partir da análise de correlação das métricas candidatas; neste caso, as métricas redundantes devem ser excluídas, de forma que as métricas centrais restantes constituam os índices multimétricos, pois métricas correlacionadas podem inflar os valores destes índices. Entretanto, avaliações das premissas para construção de índices multimétricos são raramente realizadas em sistemas distintos daqueles em que foram propostos, especialmente no Brasil.

Portanto, este estudo avaliou se havia correlação entre métricas de diferentes conjuntos de indicadores comumente usados na construção de índices multimétricos recentemente propostos. Especificamente, foi avaliado se havia correlação entre métricas que caracterizam o uso e cobertura do solo e as características da zona ripária, bem como correlações entre métricas das comunidades de macroinvertebrados e peixes, mensuradas nos mesmos pontos de amostragem em riachos numa paisagem rural no sudeste brasileiro.

\section{MATERIAL E MÉTODOS}

\section{1. Área de estudo}

Este trabalho foi desenvolvido nas bacias hidrográficas dos rios Jacaré-Pepira e Jacaré-Guaçu, ambos pertencentes à bacia hidrográfica do Tietê-Jacaré (UGRHI-13), na região central do estado de São Paulo, em riachos de primeira e segunda ordem nos 
municípios de Ibitinga, Itaju e Tabatinga. A bacia do rio Jacaré-Pepira tem área de $2.612 \mathrm{~km}^{2}$ e estende-se pelas coordenadas $21^{\circ} 55^{\prime}$ e $22^{\circ} 30^{\prime} \mathrm{S}, 47^{\circ} 55^{\prime}$ e $48^{\circ} 55^{\prime} \mathrm{W}$, sendo que o rio JacaréPepira deságua junto à represa de Ibitinga a $400 \mathrm{~m}$ de altitude. A bacia do rio Jacaré-Guaçu tem uma área de $4.108 \mathrm{~km}^{2}$ e localiza-se entre as coordenadas $21^{\circ} 37^{\prime}$ e $22^{\circ} 22^{\prime} \mathrm{S}, 47^{\circ} 43^{\prime}$ e $48^{\circ} 57^{\prime} \mathrm{W}$, sendo que o rio deságua também junto à represa de Ibitinga no rio Tietê.

O clima na região estudada é tropical quente e seco (Aw) de acordo com a classificação de Köppen, com temperaturas médias mensais entre 19,3 e 25,4 ${ }^{\circ} \mathrm{C}$ e precipitação média anual de $1.260 \mathrm{~mm}$, concentrada entre Outubro e Março. Geologicamente, ambas as bacias ocorrem nos grupos São Bento (formações Pirambóia, Botucatu e Serra Geral) e Bauru (formação Adamantina e Itaqueri, apenas na bacia do rio Jacaré-Guaçu; IPT, 2000). A UGRHI-13 está inserida numa área de transição vegetacional de Cerrado e Mata Atlântica, sendo o uso e cobertura do solo predominantemente para plantação de cana-de-açúcar e laranja, pasto e plantações de Pinus e Eucalyptus, com apenas 11,3\% de vegetação nativa remanescente; cerca de $60 \%$ da água é utilizada para irrigação e outros usos agrícolas (Tundisi et al., 2008).

\subsection{Amostragem}

A amostragem foi realizada entre 2008 e 2009, em 12 riachos selecionados para formar um gradiente de degradação da zona ripária, seis na bacia do rio Jacaré-Guaçu (Água Quente, Cana Dobrada, Jacutinga, Macaúba, Matinha, Queixada) e seis na bacia do rio Jacaré-Pepira (Bromélia, Degradado, Irara-Branca, Orquídea, Ribanceira, Ruibarbo). Em cada riacho, foi selecionado um trecho de $100 \mathrm{~m}$ para as coletas de dados, que foram caracterizados em relação ao uso e cobertura do solo a montante do final do trecho, características das zonas ripárias, e comunidades de peixes e macroinvertebrados. Os trechos foram selecionados de forma a representar homogeneamente os níveis de degradação representados por cada riacho a partir da nascente: totalmente desmatado, parcialmente desmatado, e com presença de floresta ciliar com no mínimo $30 \mathrm{~m}$ de largura.

A rede de drenagem e a topografia do terreno foram obtidas através da conversão digital de cartas topográficas na escala 1:50.000 do IBGE. A partir destas informações foi possível a delimitação de cada microbacia a partir do limite a jusante do trecho e pelos divisores de água do trecho estudado. Para caracterização do uso e cobertura do solo, de cada microbacia foi realizada interpretação visual da imagem Landsat TM-5 (221/75) de 15 de julho de 2008, em escala 1:50.000, seguindo-se procedimentos do Instituto Brasileiro de Geografia e Estatística (IBGE, 2013) com o software ArcGis 10.2.2. Foram obtidas as proporções de cobertura do solo em relação ao plantio de cana-de-açúcar, laranja, pasto, vegetação nativa, áreas urbanas e estradas. Áreas urbanas e estradas foram combinadas para se obter uma única variável (áreas urbanas) devido à baixa cobertura destas categorias.

A zona ripária e a estrutura das florestas ripárias foram descritas através de três parcelas de $100 \mathrm{~m}^{2}$ marcadas aleatoriamente no trecho estudado. Em cada parcela, foram estimadas as porcentagens de cobertura ao nível do solo por gramíneas, lianas, bambus e árvores, através das seguintes classes de cobertura: $0-25 \%, 25-50 \%, 50-75 \%, 75-100 \%$. Todos os indivíduos arbóreo-arbustivos com diâmetro a altura do peito (DAP) maiores que $5,0 \mathrm{~cm}$ foram marcados e, para cada indivíduo, foram medidos a altura total (usando um hipsômetro a laser) e estimado o DAP (a partir da circunferência de cada árvore medida com uma fita métrica). A partir destes dados, as seguintes variáveis foram estimadas para cada parcela: altura média, DAP médio, área basal total por hectare, densidade de indivíduos por hectare e estratificação da floresta (um índice relativo estimado pelo coeficiente de variação das alturas totais dos indivíduos). Os dados da zona ripária e da estrutura das florestas ripárias faziam parte de um estudo mais amplo, apresentados em Souza et al. (2013). 
Os macroinvertebrados presentes em cada trecho foram coletados usando-se amostradores tipo Surber com área de $0,09 \mathrm{~m}^{2}$ e malha de $250 \mu \mathrm{m}$. Foram amostradas aleatoriamente três unidades em cada trecho, sendo cada amostra acondicionada em sacos plásticos para triagem em laboratório. Cada amostra foi aberta em bandeja branca transiluminada, com retirada manual dos macroinvertebrados, que foram preservados em etanol $70 \%$. Os macroinvertebrados foram identificados ao nível de família, com exceção de Oligochaeta, usando-se as chaves taxonômicas de Froehlich (2007). As seguintes métricas foram então estimadas para cada amostra, representando variáveis relacionadas à composição, diversidade e tolerância à poluição dos macroinvertebrados: abundância total, riqueza de táxons, riqueza estimada por rarefação, índice de diversidade de Shannon, índice de dominância de Simpson, índice de equitatividade de Pielou, abundância relativa de EPT (Ephemeroptera, Trichoptera e Plecoptera), riqueza de táxons EPT e razão entre EPT:Chironomidae seguindo-se Rosenberg e Resh (1993). Foi estimado também o índice BMWP adaptado, seguindo-se Alba-Tercedor e Sánchez-Ortega (1988), que pondera a ocorrência das famílias em relação a sua tolerância à poluição. Estas métricas foram selecionadas considerando-se seu amplo uso no biomonitoramento de riachos em sistemas brasileiros (Baptista et al., 2007).

Os peixes foram coletados com pesca elétrica, usando-se um aparelho LR-24, Smith-Root, Inc.. Cada trecho foi delimitado com redes de malha de $5 \mathrm{~mm}$, sendo realizadas duas passagens de pesca-elétrica (tempo médio para cada riacho de $02 \mathrm{~h} 30$ horas), usando-se peneiras e puçás para captura dos peixes. Os exemplares capturados foram fixados em formol 10\%, sendo depositados na coleção de peixes do Laboratório de Ictiologia e Sistemática do Departamento de Ecologia e Biologia Evolutiva (LISDEBE) da Universidade Federal de São Carlos. Os dados deste estudo fazem parte de um estudo mais amplo de comunidades de peixes nesta região (Nassin; Oliveira; Tanaka, dados não publicados). As seguintes métricas foram estimadas para cada amostra: abundância total, riqueza de espécies, riqueza estimada por rarefação, riqueza de espécies nativas, índice de diversidade de Shannon, índice de dominância de Simpson, índice de equitatividade de Pielou, riqueza de espécies nectônicas, bentônicas, insetívoras, reofílicas, proporção de Poecilia reticulata e o índice de integridade biológica (IBI) proposto por Casatti et al. (2009), que combina a riqueza de espécies nativas, índice de dominância de Simpson, proporção de indivíduos tolerantes a hipóxia, riqueza de espécies nectônicas e riqueza de espécies reofílicas, proposto para a região oeste do estado de São Paulo.

\subsection{Análise de dados}

Como as variáveis analisadas diferem em escalas, foi usado o coeficiente de correlação não-paramétrico de Spearman para estimar a associação entre pares de variáveis, seguindo-se Sokal e Rohlf (1995). Além disso, Hering et al. (2006) sugerem que as análises de correlações entre métricas para construção de índices multimétricos sejam realizadas com o coeficiente de Spearman. O nível de significância usado no presente estudo foi $P<0,05$.

\section{RESULTADOS E DISCUSSÃO}

As microbacias dos riachos estudados apresentaram grande variação no uso e cobertura do solo, mas com predominância de usos agropecuários (Tabela 1). A cobertura máxima de vegetação natural foi de $26,8 \%$, enquanto o percentual de cobertura de culturas agrícolas foi bem mais alto, tanto para cultura de laranja (0 a 80,6\%) e cana-de-açúcar $(6,1$ a 96,0\%). No caso de cobertura por pastagem, o uso foi bem menos intenso (máximo de $24,9 \%$ ), enquanto a cobertura por estradas e estruturas urbanas juntos chegaram no máximo a 58,1\% em uma das microbacias. 
Tabela 1. Valores das métricas relacionadas ao uso do solo, composição da zona ripária e estrutura da mata ciliar no entorno de riachos numa paisagem rural no centro do estado de São Paulo. O código de cada métrica usado em tabelas posteriores também é indicado.

\begin{tabular}{|c|c|c|c|c|c|c|c|c|c|c|c|c|c|}
\hline \multicolumn{14}{|c|}{ Riachos $^{1}$} \\
\hline Métrica & Código & 1 & 2 & 3 & 4 & 5 & 6 & 7 & 8 & 9 & 10 & 11 & 12 \\
\hline \multicolumn{14}{|c|}{ Uso e cobertura do solo $(\%)$} \\
\hline Vegetação natural & VEG & 19,0 & 22,7 & 18,0 & 15,0 & 7,9 & 15,4 & 13,3 & 4,4 & 4,0 & 20,9 & 26,8 & 19,6 \\
\hline Cultura de laranja & LAR & 22,7 & 20,4 & 10,4 & 0,0 & 8,7 & 51,0 & 80,6 & 0,0 & 0,0 & 0,0 & 0,0 & 7,8 \\
\hline $\begin{array}{l}\text { Cultura de cana- } \\
\text { de-açúcar }\end{array}$ & CAN & 58,3 & 56,9 & 62,0 & 60,1 & 80,3 & 33,6 & 6,1 & 28,1 & 96,0 & 79,1 & 73,2 & 72,4 \\
\hline Pasto & PAS & 0,0 & 0,0 & 9,5 & 24,9 & 0,0 & 0,0 & 0,0 & 9,4 & 0,0 & 0,0 & 0,0 & 0,0 \\
\hline Urbano & URB & 0,0 & 0,0 & 0,0 & 0,0 & 3,1 & 0,0 & 0,0 & 58,1 & 0,0 & 0,0 & 0,0 & 0,0 \\
\hline \multicolumn{14}{|c|}{ Zona ripária e mata ciliar } \\
\hline \multicolumn{14}{|l|}{ (\%) de Cobertura: } \\
\hline Gramíneas & GRA & 25 & 25 & 100 & 100 & 100 & 92 & 25 & 100 & 25 & 25 & 25 & 58 \\
\hline Bambu & BAM & 25,0 & 25,0 & 25,0 & 25,0 & 25,0 & 25,0 & 25,0 & 62,5 & 25,0 & 25,0 & 25,0 & 25,0 \\
\hline Lianas & LIA & 50,0 & 58,3 & 25,0 & 25,0 & 25,0 & 25,0 & 25,0 & 25,0 & 25,0 & 33,3 & 91,7 & 41,7 \\
\hline Árvores & ARV & 58,3 & 83,3 & 25,0 & 25,0 & 25,0 & 50,0 & 83,3 & 25,0 & 91,7 & 75,0 & 66,7 & 50,0 \\
\hline $\begin{array}{l}\text { Largura da mata } \\
\text { ciliar }(\mathrm{m})\end{array}$ & LARG & 13,7 & 50,0 & 0,0 & 0,0 & 0,0 & 5,0 & 38,3 & 0,0 & 40,0 & 16,0 & 19,8 & 6,9 \\
\hline $\begin{array}{l}\text { Altura das } \\
\text { árvores (m) }\end{array}$ & ALT & 7,7 & 6,7 & 0,0 & 0,0 & 0,0 & 6,3 & 7,3 & 3,1 & 11,2 & 8,1 & 5,5 & 6,1 \\
\hline $\begin{array}{l}\text { Diâmetro a altura } \\
\text { do peito }(\mathrm{cm})\end{array}$ & DAP & 14,1 & 12,5 & 0,0 & 0,0 & 0,0 & 15,3 & 10,1 & 6,1 & 29,4 & 11,8 & 11,2 & 12,9 \\
\hline $\begin{array}{l}\text { Área basal } \\
\left(\mathrm{m}^{2} \mathrm{ha}^{-1}\right)\end{array}$ & $\mathrm{AB}$ & 27,8 & 25,5 & 0,0 & 0,0 & 0,0 & 11,3 & 42,3 & 0,2 & 85,8 & 37,1 & 14,5 & 12,3 \\
\hline $\begin{array}{l}\text { Densidade } \\
\left(\text { ind }^{-1}{ }^{-1} \text { ) }\right.\end{array}$ & DENS & 1433,3 & 1166,7 & 0,0 & 0,0 & 0,0 & 400,0 & 4233,3 & 66,7 & 833,3 & 2600,0 & 1233,3 & 833,3 \\
\hline $\begin{array}{l}\text { Estratificação } \\
\text { da copa }\end{array}$ & ESTR & 0,29 & 0,40 & 0,00 & 0,00 & 0,00 & 0,37 & 0,30 & 0,19 & 0,56 & 0,31 & 0,42 & 0,35 \\
\hline
\end{tabular}

1 Riachos amostrados: 1 - Água Quente; 2 - Bromélia; 3 - Cana Dobrada; 4 - Degradado; 5 - Irara Branca; 6 - Jacutinga; 7 -Macaúba; 8 - Matinha; 9 - Orquídea; 10 - Queixada; 11 - Ribanceira; 12 - Ruibarbo.

A zona ripária variou de um gradiente de $100 \%$ de cobertura de gramíneas a 91,7\% de cobertura por árvores, sendo que as florestas ripárias apresentaram grande variação em sua estrutura, com densidade variando entre 67 e 4.233 ind ha $^{-1}$, e área basal total variando entre 0,2 e $85,8 \mathrm{~m}^{2} \mathrm{ha}^{-1}$ (Tabela 1). Estas diferenças na zona ripária e uso do solo resultaram em grande variação nas comunidades aquáticas. A abundância de macroinvertebrados variou entre 40 e 365 , enquanto a riqueza de táxons variou entre 6 e 29; por outro lado, o número total de peixes amostrados variou de 0 a 303, enquanto a riqueza de espécies variou de 0 a 9 , pois não foram observados peixes no córrego Degradado (Tabela 2).

Não houve correlação significativa entre as métricas de uso e cobertura do solo (Tabela 3). Destas, apenas a proporção de pasto na microbacia foi negativamente correlacionada com a largura das florestas ripárias, e também com indicadores de estrutura da floresta (Tabela 3). Isto sugere que, na região estudada, os riachos que ocorreram em áreas com pouca ou nenhuma floresta ripária estavam localizados predominantemente em área de 
pastagens. Atividades pecuárias são importantes condutores de degradação de zonas ripárias em regiões tropicais devido ao desmatamento e ao acesso do gado à água (Neill et al., 2001), com fortes efeitos nas comunidades aquáticas e na própria estrutura dos riachos (Lorion e Kennedy, 2009). O desmatamento para o estabelecimento de pastos pode chegar até a beira dos riachos, muitas vezes com maiores efeitos de desmatamento em pequenas propriedades que em grandes propriedades (Michalski et al., 2010). De fato, Wyman e Stein (2010) verificaram que a pecuária foi o principal condutor do desmatamento e degradação das florestas ripárias num estudo em Belize, encontrando maior probabilidade de desmatamento em áreas mais próximas ao rio. Desta forma, é possível que diferentes práticas agrícolas (pecuária, culturas anuais, culturas perenes) tenham efeitos distintos na relação entre o uso e cobertura do solo na bacia e as características das zonas ripárias, mas mais estudos são necessários para testar esta hipótese.

Tabela 2. Valores das métricas biológicas relacionadas à composição e estrutura de comunidades de macroinvertebrados e peixes em riachos numa paisagem rural no centro do estado de São Paulo. O código de cada métrica usado em tabelas posteriores também é indicado.

\begin{tabular}{|c|c|c|c|c|c|c|c|c|c|c|c|c|c|}
\hline \multirow[b]{2}{*}{ Métrica } & \multicolumn{13}{|c|}{ Riachos $^{1}$} \\
\hline & Código & 1 & 2 & 3 & 4 & 5 & 6 & 7 & 8 & 9 & 10 & 11 & 12 \\
\hline & \multicolumn{13}{|c|}{ Macroinvertebrados } \\
\hline Abundância total & $\mathrm{Nm}$ & 71 & 169 & 59 & 264 & 126 & 365 & 86 & 40 & 356 & 82 & 330 & 125 \\
\hline Riqueza de táxons & $\mathrm{Sm}$ & 6 & 16 & 13 & 17 & 22 & 18 & 9 & 6 & 29 & 7 & 17 & 18 \\
\hline Riqueza de táxons por rarefação & $\mathrm{E}(\mathrm{S}) \mathrm{m}$ & 4,5 & 5,8 & 5,3 & 6,1 & 11,3 & 5,0 & 5,0 & 4,0 & 11,2 & 3,2 & 4,6 & 8,1 \\
\hline Índice de diversidade de Shannon & H'm & 1,19 & 1,45 & 1,28 & 1,37 & 2,11 & 1,15 & 0,60 & 0,90 & 2,22 & 0,55 & 0,85 & 1,69 \\
\hline Índice de dominância de Simpson & Dm & 0,37 & 0,31 & 0,45 & 0,36 & 0,20 & 0,43 & 0,77 & 0,50 & 0,17 & 0,77 & 0,63 & 0,27 \\
\hline Índice de equitatividade de Pielou & $J^{\prime} m$ & 0,66 & 0,52 & 0,50 & 0,48 & 0,68 & 0,40 & 0,27 & 0,50 & 0,66 & 0,28 & 0,30 & 0,59 \\
\hline BMWP & BMWP & 20 & 72 & 60 & 81 & 116 & 99 & 49 & 22 & 165 & 28 & 83 & 99 \\
\hline Abundância relativa de EPT & $\%$ EPT & 0,00 & 0,53 & 0,05 & 0,12 & 0,12 & 0,07 & 0,02 & 0,00 & 0,42 & 0,00 & 0,01 & 0,04 \\
\hline Riqueza taxonômica de EPT & SEPT & 1 & 3 & 4 & 4 & 5 & 5 & 2 & 0 & 10 & 0 & 4 & 6 \\
\hline \multirow[t]{2}{*}{ EPT/Chironomidae } & EPT/Chi & 0,01 & 1,33 & 0,08 & 0,37 & 0,33 & 0,23 & 0,02 & 0,00 & 1,38 & 0,00 & 0,01 & 0,10 \\
\hline & \multicolumn{13}{|c|}{ Peixes } \\
\hline Abundância total & $\mathrm{Np}$ & 180 & 135 & 67 & 0 & 115 & 111 & 39 & 225 & 303 & 14 & 74 & 25 \\
\hline Riqueza de espécies & $\mathrm{Sp}$ & 2 & 7 & 8 & 0 & 8 & 5 & 1 & 9 & 3 & 3 & 8 & 9 \\
\hline Riqueza de espécies por rarefação & $E(S) p$ & 1,5 & 4,1 & 5,1 & 0,0 & 5,1 & 3,3 & 1,0 & 5,2 & 1,5 & 3,0 & 5,3 & 6,8 \\
\hline Riqueza de espécies nativas & $\mathrm{SNp}$ & 2,0 & 6,0 & 8,0 & 0,0 & 7,0 & 4,0 & 1,0 & 8,0 & 3,0 & 3,0 & 7,0 & 9,0 \\
\hline Índice de diversidade de Shannon & H'p & 0,20 & 1,21 & 1,59 & & 1,54 & 1,13 & 0,00 & 1,73 & 0,18 & 0,89 & 1,57 & 1,88 \\
\hline Índice de dominância de Simpson & $\mathrm{Dp}$ & 0,9 & 0,4 & 0,3 & & 0,3 & 0,4 & 1,0 & 0,2 & 0,9 & 0,5 & 0,3 & 0,2 \\
\hline Índice de equitatividade de Pielou & $J^{\prime} p$ & 0,29 & 0,62 & 0,77 & 0,00 & 0,74 & 0,70 & 0,00 & 0,79 & 0,16 & 0,81 & 0,76 & 0,86 \\
\hline Riqueza de espécies nectônicas & Snec & 0 & 2 & 3 & 0 & 3 & 2 & 0 & 4 & 0 & 0 & 3 & 3 \\
\hline Riqueza de espécies bentônicas & Sben & 1 & 4 & 5 & 0 & 3 & 1 & 0 & 3 & 1 & 1 & 4 & 5 \\
\hline Riqueza de espécies insetívoras & Sins & 1 & 4 & 5 & 0 & 2 & 2 & 0 & 3 & 2 & 2 & 5 & 4 \\
\hline Riqueza de espécies reofílicas & Sreo & 0 & 0 & 2 & 0 & 0 & 0 & 0 & 1 & 0 & 0 & 1 & 1 \\
\hline $\begin{array}{l}\text { Abundância relativa de } P \text {, } \\
\text { reticulata }\end{array}$ & $\% \mathrm{POE}$ & 0,0 & 13,3 & 0,0 & 0,0 & 1,7 & 0,9 & 0,0 & 28,4 & 1,7 & 14,3 & 0,0 & 0,0 \\
\hline Índice de Integridade Biológica & IBI & 1,8 & 1,4 & 2,6 & 0,0 & 1,8 & 1,4 & 1,8 & 2,2 & 1,8 & 1,8 & 2,2 & 2,6 \\
\hline
\end{tabular}

${ }^{1}$ Riachos amostrados: 1 - Água Quente; 2 - Bromélia; 3 - Cana Dobrada; 4 - Degradado; 5 - Irara Branca; 6 - Jacutinga; 7 - Macaúba; 8 - Matinha; 9 - rquídea; 10 - Queixada; 11 - Ribanceira; 12 - Ruibarbo. 
Dos indicadores da zona ripária, a proporção de árvores foi correlacionada positivamente com a largura da mata ciliar e também com os indicadores de estrutura da floresta, enquanto a proporção de gramíneas foi negativamente correlacionada com a cobertura por lianas, árvores, com a largura da mata ciliar e com todos os indicadores de estrutura da floresta (Tabela 3). Nos trechos com mata ciliar, todos os indicadores de estrutura da floresta foram correlacionados positivamente entre si, com exceção da densidade, que não foi correlacionada com o DAP nem com a estratificação (Tabela 3). A densidade de árvores é determinada pelo estágio seral das florestas e também por respostas locais à frequência e intensidade de perturbações (Fernandes et al., 2014). Por exemplo, Balian e Naiman (2005) encontraram redução de 26.000 para 500 ramos por hectare durante o desenvolvimento inicial de florestas pluviais costeiras da América do Norte. Souza et al. (2013) verificaram que um importante descritor multivariado da estrutura de florestas ripárias era um eixo que separava áreas com florestas de alta densidade e baixo DAP daquelas com baixa densidade e alto DAP, com fortes efeitos na condutividade elétrica e concentrações de nutrientes na água. Fernandes et al. (2014) verificaram que, num grande fragmento de floresta ripária, o descritor multivariado da estrutura da floresta que separava trechos com alta densidade e baixa área basal daqueles com baixa densidade e alta área basal, também influenciava as concentrações de nutrientes no riacho. A densidade de árvores é um importante componente da estrutura das florestas, que por sua vez pode influenciar os ciclos hidrológicos e o deslocamento de nutrientes para os riachos (Dosskey et al., 2010, Souza et al., 2013). Assim, mesmo para uma dada cobertura de florestas ripárias numa microbacia, tais florestas podem apresentar diferenças em sua estrutura.

Tabela 3. Valores do coeficiente de correlação de Spearman calculado entre métricas indicadoras do uso do solo, composição da zona ripária e estrutura da mata ciliar em riachos de uma paisagem rural do sudeste brasileiro.

\begin{tabular}{|c|c|c|c|c|c|c|c|c|c|c|c|c|c|c|}
\hline & ALT & DAP & $\mathrm{AB}$ & DENS & ESTR & GRA & BAM & LIA & ARV & LARG & VEG & LAR & PAS & URB \\
\hline DAP & 0,794 & & & & & & & & & & & & & \\
\hline $\mathrm{AB}$ & 0,950 & 0,674 & & & & & & & & & & & & \\
\hline DENS & 0,789 & 0,480 & 0,867 & & & & & & & & & & & \\
\hline ESTR & 0,652 & 0,787 & 0,681 & 0,526 & & & & & & & & & & \\
\hline GRA & $-0,855$ & $-0,637$ & $-0,924$ & $-0,913$ & $-0,733$ & & & & & & & & & \\
\hline BAM & $-0,220$ & $-0,220$ & $-0,220$ & $-0,220$ & $-0,220$ & 0,381 & & & & & & & & \\
\hline LIA & 0,275 & 0,330 & 0,322 & 0,547 & 0,479 & $-0,616$ & $-0,243$ & & & & & & & \\
\hline ARV & 0,871 & 0,644 & 0,943 & 0,795 & 0,788 & $-0,934$ & $-0,357$ & 0,382 & & & & & & \\
\hline LARG & 0,789 & 0,609 & $\mathbf{0 , 8 8 2}$ & 0,779 & 0,810 & $-0,934$ & $-0,356$ & 0,516 & 0,978 & & & & & \\
\hline VEG & 0,092 & 0,141 & 0,106 & 0,423 & 0,317 & $-0,430$ & $-0,393$ & 0,866 & 0,211 & 0,331 & & & & \\
\hline LAR & 0,131 & 0,168 & 0,095 & 0,223 & $-0,073$ & $-0,130$ & $-0,317$ & $-0,049$ & 0,122 & 0,137 & 0,051 & & & \\
\hline PAS & $-0,656$ & $-0,656$ & $-0,656$ & $-0,657$ & $-0,656$ & 0,720 & 0,401 & $-0,461$ & $-0,675$ & $-0,673$ & $-0,275$ & $-0,314$ & & \\
\hline URB & $-0,444$ & $-0,444$ & $-0,444$ & $-0,445$ & $-0,444$ & 0,562 & 0,739 & $-0,360$ & $-0,527$ & $-0,525$ & $-0,521$ & $-0,223$ & 0,205 & \\
\hline CAN & 0,056 & 0,063 & 0,049 & $-0,148$ & 0,169 & $-0,080$ & $-0,393$ & 0,117 & 0,086 & 0,050 & 0,049 & $-0,566$ & $-0,211$ & $-0,048$ \\
\hline
\end{tabular}

Nota: Valores em negrito indicam $\mathrm{P}<0,05$. Códigos como na Tabela 1. 
Neste estudo, não foi encontrada redundância entre as métricas de uso e cobertura do solo, e houve pouca correlação entre estes indicadores e métricas da zona ripária, sugerindo que informações tanto sobre o uso e cobertura do solo quanto da composição da zona ripária e estrutura das florestas podem ser importantes para o monitoramento de riachos. Esta influência conjunta é reconhecida na avaliação ambiental de riachos (Goforth e Bain, 2010). Por exemplo, Petersen (1992) propôs o índice RCE (Riparian, Channel, and Environmental Inventory), que combina métricas de uso do solo, composição da zona ripária, qualidade do habitat do canal, e composição das comunidades aquáticas, a partir de informações de campo.

As comunidades aquáticas apresentaram as maiores correlações entre as métricas biológicas, apesar de haver algumas diferenças entre os dois grupos estudados. Na construção de índices multimétricos, Hering et al. (2006) sugerem que a seleção das métricas centrais deve considerar apenas variáveis não correlacionadas entre si, para evitar redundância das informações nos valores finais dos índices. Para as comunidades de macroinvertebrados, quase todos os indicadores apresentaram correlações significativas entre si (Tabela 4). $\mathrm{O}$ índice de equitatividade de Pielou foi positivamente correlacionado com o índice de diversidade de Shannon e negativamente correlacionado com o índice de dominância de Simpson (Tabela 4). Outra exceção foi a abundância total, que não foi correlacionada com nenhum índice de dominância, diversidade ou equitatividade, mas apresentou correlação positiva com a riqueza de táxons. Ao retirar o efeito da amostragem usando-se rarefação, a estimativa da riqueza deixou de ser correlacionada com a abundância total (Tabela 4).

Tabela 4. Valores do coeficiente de correlação de Spearman calculado entre métricas indicadoras das comunidades de macroinvertebrados em riachos de uma paisagem rural do sudeste brasileiro.

\begin{tabular}{llllllllll}
\hline & $\mathrm{Nm}$ & $\mathrm{Sm}$ & $\mathrm{E}(\mathrm{S}) \mathrm{m}$ & $\mathrm{H}$ 'm & $\mathrm{Dm}$ & $\mathrm{J}$ 'm & \%EPT & SEPT & EPT/Chiro \\
\hline $\mathrm{Sm}$ & $\mathbf{0 , 7 8 4}$ & & & & & & & & \\
E(S)m & 0,483 & $\mathbf{0 , 8 4 4}$ & & & & & & & \\
H'm & 0,322 & $\mathbf{0 , 6 8 9}$ & $\mathbf{0 , 9 0 2}$ & & & & & & \\
Dm & $-0,392$ & $\mathbf{- 0 , 6 8 9}$ & $\mathbf{- 0 , 8 3 9}$ & $\mathbf{- 0 , 9 7 2}$ & & & & & \\
J'm & $-0,049$ & 0,306 & 0,545 & $\mathbf{0 , 8 1 1}$ & $\mathbf{- 0 , 8 3 9}$ & & & & \\
\%EPT & $\mathbf{0 , 6 2 0}$ & $\mathbf{0 , 7 3 1}$ & $\mathbf{0 , 8 5 8}$ & $\mathbf{0 , 7 8 1}$ & $\mathbf{- 0 , 7 3 6}$ & 0,389 & & & \\
SEPT & $\mathbf{0 , 6 7 1}$ & $\mathbf{0 , 9 4 9}$ & $\mathbf{0 , 8 4 5}$ & $\mathbf{0 , 7 3 1}$ & $\mathbf{- 0 , 7 0 3}$ & 0,371 & $\mathbf{0 , 6 7 6}$ & & \\
EPT/Chiro & $\mathbf{0 , 6 8 3}$ & $\mathbf{0 , 7 8 0}$ & $\mathbf{0 , 8 7 9}$ & $\mathbf{0 , 8 0 2}$ & $\mathbf{- 0 , 7 7 1}$ & 0,375 & $\mathbf{0 , 9 7 9}$ & $\mathbf{0 , 7 3 6}$ & \\
BMWP & $\mathbf{0 , 7 7 8}$ & $\mathbf{0 , 9 9 6}$ & $\mathbf{0 , 8 2 0}$ & $\mathbf{0 , 6 6 2}$ & $\mathbf{- 0 , 6 5 8}$ & 0,284 & $\mathbf{0 , 7 0 5}$ & $\mathbf{0 , 9 4 0}$ & $\mathbf{0 , 7 5 1}$ \\
\hline
\end{tabular}

Nota: Valores em negrito indicam $\mathrm{P}<0,05$. Códigos como na Tabela 2.

Estas métricas são comumente usadas na construção de índices multimétricos, mas apresentaram correlações significativas entre si, indicando redundância das informações na região estudada. Por exemplo, no caso de riachos brasileiros de baixa ordem, Baptista et al. (2007) propuseram um índice para riachos de floresta atlântica composto pela proporção de Diptera e Coleoptera, riqueza de famílias, riqueza de gêneros de EPT, BMWP e porcentagem de fragmentadores; Couceiro et al. (2012) propuseram um índice para aplicação em riachos na Amazônia Central composto por riqueza de famílias, riqueza de gêneros de EPT, proporção de EPT, EPT/Chironomidae, riqueza de táxons sensíveis, proporção de coletores e 
fragmentadores; Suriano et al. (2011) propuseram as seguintes variáveis como candidatas para compor um índice multimétrico para avaliar o efeito de diferentes usos do solo em riachos no estado de São Paulo: riqueza de famílias, riqueza de famílias de EPT, proporção de EPT, Megaloptera, Hirudinea, índice de diversidade de Shannon estimado para gêneros e BMWP. Todos estes estudos testaram correlações entre as métricas propostas, não encontrando resultados significativos, mas em nosso estudo fortes correlações foram encontradas entre a riqueza estimada por rarefação e a proporção de EPT, riqueza de famílias EPT, EPT/Chironomidae e BMWP, além dos índices de diversidade de Shannon e dominância de Simpson.

As diferenças entre os resultados encontrados por estes autores e os resultados do presente estudo podem ser devido ao desenho de amostragem dos riachos, pois para a construção destes índices, estes autores selecionaram riachos em categorias extremas (preservados vs. impactados). As respostas de comunidades e indicadores biológicos ao estresse ambiental podem ser lineares ou não-lineares, sendo que, neste último caso, as respostas previstas pelos modelos podem ser extremamente diferentes (Davies e Jackson, 2006) e, portanto, ao se amostrar apenas categorias extremas, não há como prever as respostas em níveis intermediários de estresse ambiental. Os riachos estudados representavam um gradiente de impactos por atividades rurais, incluindo não apenas as categorias extremas como também os níveis intermediários, possivelmente resultando na correlação entre as variáveis estudadas. De fato, a amostragem dos níveis intermediários de estresse ambiental é fortemente recomendada por Hering et al. (2006) na construção de índices multimétricos. Além disso, no desenvolvimento dos índices, os riachos considerados impactados estavam sujeitos a diferentes condutores de degradação. O tipo de cobertura do solo pode resultar em respostas distintas das comunidades biológicas, devido a diferentes efeitos nos padrões de retenção de nutrientes e poluentes, erosão, sedimentação, entre outros (Miserendino et al., 2011).

A única variável não correlacionada com indicadores de riqueza e composição de macroinvertebrados foi o índice de equitatividade de Pielou. Indicadores de diversidade podem apresentar variação em diferentes escalas espaciais; por exemplo, Boyero e Bailey (2001) encontraram diferenças entre riachos de diferentes ordens nas estimativas de riqueza de táxons, mas não na estimativa da equitatividade. Em outro estudo, Boyero (2003) encontrou variação em escalas espaciais pequenas na riqueza de táxons (entre amostras dentro de seções de corredeiras), enquanto a equitatividade variou em escalas espaciais médias (entre corredeiras dentro de segmentos, e entre segmentos de riachos). Assim, a ausência de correlação significativa entre a equitatividade e os demais indicadores da estrutura e composição das comunidades de macroinvertebrados pode resultar da variação em diferentes escalas espaciais destes indicadores.

Nas comunidades de peixes, a abundância total não foi correlacionada com nenhuma outra métrica, assim como a proporção de Poecilia reticulata nos riachos (Tabela 5). As demais métricas foram significativamente correlacionadas entre si, com correlações negativas em relação ao índice de dominância de Simpson, e positivas para todo o restante (Tabela 5).

$\mathrm{Na}$ proposição de um índice biológico para o oeste do estado de São Paulo, na bacia do Alto Paraná, Casatti et al. (2009) sugeriram que as seguintes métricas fossem incluídas: riqueza de espécies nativas, índice de dominância de Simpson, riqueza de espécies nectônicas, reofílicas e proporção de indivíduos tolerantes a hipóxia. No presente estudo, todas as variáveis relacionadas à riqueza e diversidade foram fortemente correlacionadas entre si, sugerindo a necessidade de adaptar o índice para a região estudada. Por outro lado, a abundância total não foi correlacionada com estas variáveis. 
Tabela 5. Valores do coeficiente de correlação de Spearman calculado entre métricas indicadoras das comunidades de peixes em riachos de uma paisagem rural do sudeste brasileiro.

\begin{tabular}{lllllllllllll}
\hline & Np & Sp & E(S)p & SNp & H'p & Dp & J'p & Snec & Sben & Sins & Sreo & \%POE \\
\hline Sp & 0,226 & & & & & & & & & & & \\
E(S)p & 0,098 & $\mathbf{0 , 9 6 1}$ & & & & & & & & & & \\
SNp & 0,162 & $\mathbf{0 , 9 8 6}$ & $\mathbf{0 , 9 5 6}$ & & & & & & & & & \\
H'p & 0,116 & $\mathbf{0 , 9 7 7}$ & $\mathbf{0 , 9 7 7}$ & $\mathbf{0 , 9 8 2}$ & & & & & & & & \\
Dp & 0,155 & $\mathbf{- 0 , 9 6 3}$ & $\mathbf{- 0 , 9 6 4}$ & $\mathbf{- 0 , 9 7 0}$ & $\mathbf{- 0 , 9 9 1}$ & & & & & & & \\
J'p & $-0,137$ & $\mathbf{0 , 7 9 6}$ & $\mathbf{0 , 8 2 3}$ & $\mathbf{0 , 8 0 6}$ & $\mathbf{0 , 8 4 6}$ & $\mathbf{- 0 , 8 0 9}$ & & & & & & \\
Snec & 0,185 & $\mathbf{0 , 9 4 5}$ & $\mathbf{0 , 9 0 7}$ & $\mathbf{0 , 9 2 1}$ & $\mathbf{0 , 9 2 7}$ & $\mathbf{- 0 , 9 2 7}$ & $\mathbf{0 , 6 6 4}$ & & & & & \\
Sben & 0,122 & $\mathbf{0 , 8 7 0}$ & $\mathbf{0 , 9 0 4}$ & $\mathbf{0 , 9 1 5}$ & $\mathbf{0 , 9 0 5}$ & $\mathbf{- 0 , 8 2 9}$ & $\mathbf{0 , 7 0 4}$ & $\mathbf{0 , 7 8 6}$ & & & & \\
Sins & 0,132 & $\mathbf{0 , 8 1 9}$ & $\mathbf{0 , 8 6 6}$ & $\mathbf{0 , 8 5 4}$ & $\mathbf{0 , 8 4 2}$ & $\mathbf{- 0 , 7 5 7}$ & $\mathbf{0 , 6 8 1}$ & $\mathbf{0 , 7 4 7}$ & $\mathbf{0 , 9 3 6}$ & & & \\
Sreo & $-0,071$ & $\mathbf{0 , 7 4 3}$ & $\mathbf{0 , 7 8 2}$ & $\mathbf{0 , 7 8 8}$ & $\mathbf{0 , 8 0 0}$ & $\mathbf{- 0 , 8 1 0}$ & $\mathbf{0 , 6 4 4}$ & $\mathbf{0 , 7 6 3}$ & $\mathbf{0 , 7 5 0}$ & $\mathbf{0 , 7 7 2}$ & & \\
\%POE & 0,324 & 0,150 & $-0,038$ & 0,048 & 0,038 & $-0,010$ & 0,200 & 0,095 & $-0,133$ & $-0,054$ & $-0,291$ & \\
IBI & 0,026 & $\mathbf{0 , 7 0 0}$ & $\mathbf{0 , 7 2 6}$ & $\mathbf{0 , 7 3 9}$ & $\mathbf{0 , 7 2 3}$ & $\mathbf{- 0 , 6 5 8}$ & $\mathbf{0 , 6 8 3}$ & $\mathbf{0 , 6 2 0}$ & $\mathbf{0 , 6 7 7}$ & $\mathbf{0 , 6 3 3}$ & $\mathbf{0 , 8 5 9}$ & $-0,368$ \\
\hline
\end{tabular}

Nota: Valores em negrito indicam $\mathrm{P}<0,05$. Códigos como na Tabela 2.

Em um estudo mais amplo na mesma região, Nassin, Oliveira e Tanaka (dados não publicados) avaliaram a influência das características dos riachos nas comunidades de peixes e verificaram que o volume de água no trecho estudado influenciou os indicadores de riqueza e diversidade das comunidades, enquanto a abundância total foi influenciada apenas pela condutividade elétrica. Portanto, os fatores ambientais que influenciam a riqueza e diversidade de comunidades de peixes são diferentes dos fatores que influenciam a abundância total. Assim, a abundância total de peixes pode vir a ser uma variável indicadora importante da qualidade da água dos riachos nesta região, conforme já sugerido para outras regiões (e.g., Angermeier e Karr, 1986).

\section{CONCLUSÃO}

O presente estudo mostrou que diversas métricas comumente incluídas na construção de índices multimétricos e na análise da influência das características das microbacias na qualidade ambiental de riachos podem apresentar redundância, ainda que nos estudos originais em que estes índices foram propostos estas variáveis não fossem correlacionadas. Os resultados deste estudo sugerem que o desenvolvimento de índices deve ser bastante específico para cada região estudada, e que muitas vezes as avaliações são feitas em relação a condutores de impactos específicos, que podem variar entre diferentes sistemas. As respostas das variáveis biológicas podem variar em relação a diferentes tipos de estressores e, portanto, é necessário compreender melhor as respostas dos indicadores biológicos a diferentes tipos de condutores de impactos, em especial a gradientes de impactos, para modelar as respostas esperadas destes indicadores biológicos.

\section{AGRADECIMENTOS}

Agradecemos ao Sindicato Rural de Ibitinga pelo apoio e contatos com os proprietários rurais, ao Conselho Nacional de Desenvolvimento Científico e Tecnológico (CNPq, 
Procs. 552554/2007-3 e 302890/2007-6) e Fundação de Amparo à Pesquisa do Estado de São Paulo (FAPESP, Proc. 2007/02326-0) pelo apoio financeiro e a todos que ajudaram nos trabalhos de campo.

\section{REFERÊNCIAS}

ALBA-TERCEDOR, J.; SÁNCHEZ-ORTEGA, A. Un método rápido y simple para evaluar la calidad biológica de las aguas corrientes basado en el de Hellawell (1978). Limnetica, Madrid, v. 4, p. 51-56, 1988.

ANGERMEIER, P. L.; KARR, J. R. Applying an index of biotic integrity based on streamfish communities: considerations in sampling and interpretation. North American Journal of Fisheries Management, v. 6, n. 3, p. 418-429, 1986. http://dx.doi.org/10.1577/1548-8659(1986)6<418:AAIOBI>2.0.CO;2

BALIAN, E. V.; NAIMAN, R. J. Abundance and production of riparian trees in the lowland floodplain of the Queets River, Washington. Ecosystems, New York, v. 8, n. 7, p. 841861, 2005. http://dx.doi.org/10.1007/s10021-005-0043-4

BAPTISTA, D. F.; BUSS, D. F.; EGLER, M.; GIOVANELLI, A.; SILVEIRA, M. P.; NESSIMIAN, J. L. A multimetric index based on benthic macroinvertebrates for evaluation of Atlantic Forest streams at Rio de Janeiro State, Brazil. Hydrobiologia, v. 575, n. 1, p. 83-94, 2007. http://dx.doi.org/10.1007/s10750-006-0286-x

BARBOUR, M. T.; GERRITSEN, J.; SNYDER, B. D.; STRIBLING, J. B. Rapid bioassessment protocols for use in streams and wadeable rivers: periphyton, benthic macroinvertebrates and fish. 2nd Edition. EPA 841-B-99-002. Washington, D.C.: U.S. Environmental Protection Agency, 1999.

BOYERO, L. Multiscale patterns of spatial variation in stream macroinvertebrate communities. Ecological Research, v. 18, n. 4, p. 365-379, 2003. http://dx.doi.org/10.1046/j.1440-1703.2003.00562.x

BOYERO, L.; BAILEY, R. C. Organization of macroinvertebrate communities at a hierarchy of spatial scales in a tropical stream. Hydrobiologia, v. 464, n. 1-3, p. 219-225, 2001. http://dx.doi.org/10.1023/A:1013922307096

BRAUN, D. C.; REYNOLDS, J. D. Cost-effective variable selection in habitat surveys. Methods in Ecology and Evolution, London, v. 3, n. 2, p. 388-396, 2012. http://dx.doi.org/10.1111/j.2041-210X.2011.00154.x

BUSS, D. F.; CARLISLE, D. M.; CHON, T. S.; CULP, J.; HARDING, J. S.; KEIZERVLEK, H. E. et al. Stream biomonitoring using macroinvertebrates around the globe: a comparison of large-scale programs. Environmental monitoring and assessment, v. 187, n. 1, p. 1-21, 2015. http://dx.doi.org/10.1007/s10661-014-4132-8

CASATTI, L.; FERREIRA, C. P.; LANGEANI, F. A fish-based biotic integrity index for assessment of lowland streams in southeastern Brazil. Hydrobiologia, v. 623, n. 1, p. 173-189, 2009. http://dx.doi.org/10.1007/s10750-008-9656-x

CAUGHLAN, L.; OAKLEY, K. L. Cost considerations for long-term ecological monitoring. Ecological Indicators, v. 1, n. 2, p. 123-134, 2001. http://dx.doi.org/10.1016/S1470160X(01)00015-2 
CLAPCOTT, J. E.; COLLIER, K. J.; DEATH, R. G.; GOODWIN, E. O.; HARDING, J. S.; KELLY, D. et al. Quantifying relationships between land-use gradients and structural and functional indicators of stream ecological integrity. Freshwater Biology, v. 57, n. 1, p. 74-90, 2012. http://dx.doi.org/10.1111/j.1365-2427.2011.02696.x

COUCEIRO, S. R. M.; HAMADA, N.; FORSBERG, B. R.; PIMENTEL, T. P.; LUZ, S. L. B. A macroinvertebrate multimetric index to evaluate the biological condition of streams in the Central Amazon region of Brazil. Ecological Indicators, v. 18, p. 118-125, 2012. http://dx.doi.org/10.1016/j.ecolind.2011.11.001

DAVIES, S. P.; JACKSON, S. K. The biological condition gradient: a descriptive model for interpreting change in aquatic ecosystems. Ecological Applications, v. 16, n. 4, p. 1251-1266, 2006.

http://dx.doi.org/10.1890/1051-0761(2006)016[1251:TBCGAD]2.0.CO;2

DOSSKEY, M. G.; VIDON, P.; GURWICK, N. P.; ALLAN, C. J.; DUVAL, T. P.; LOWRANCE, $\mathrm{R}$. The role of riparian vegetation in protecting and improving chemical water quality in streams. Journal of the American Water Resources Association, v. 46, n. 2, p. 261-277, 2010. http://dx.doi.org/10.1111/j.1752-1688.2010.00419.x

FERNANDES, J. F.; SOUZA, A. L. T.; TANAKA, M. O. Can the structure of a riparian forest remnant influence stream water quality? A tropical case study. Hydrobiologia, v. 724, n. 1, p. 175-185, 2014. http://dx.doi.org/10.1007/s10750-013-1732-1

FRIBERG, N.; BONADA, N.; BRADLEY, D. C.; DUNBAR, M. J.; EDWARDS, F. K.; GREY, J. et al. Biomonitoring of human impacts in freshwater ecosystems: the good, the bad and the ugly. Advances in Ecological Research, v. 44, p. 1-68, 2011. http://dx.doi.org/10.1016/B978-0-12-374794-5.00001-8

FROEHLICH, C. G. (org.). Guia on-line: identificação de larvas de insetos aquáticos do Estado de São Paulo. 2007. Disponível em:

http://sites.ffclrp.usp.br/aguadoce/Guia_online. Acesso em: 11 mar. 2015.

GOFORTH, R. R.; BAIN, M. B. Assessing stream integrity based on interpretations of mapbased riparian and subbasin properties. Landscape and Ecological Engineering, v. 8, n. 1, p. 33-43, 2010. http://dx.doi.org/10.1007/s11355-010-0138-8

HERING, D.; FELD, C. K.; MOOG, O.; OFENBÖCK, T. Cook book for the development of a Multimetric Index for biological condition of aquatic ecosystems: experiences from the European AQEM and STAR projects and related initiatives. Hydrobiologia, v. 566, n. 1, p. 311-324, 2006. http://dx.doi.org/10.1007/s10750-006-0087-2

INSTITUTO BRASILEIRO DE GEOGRAFIA E ESTATÍSTICA. Manuais técnicos em geociências: manual técnico de uso da terra. 3. ed. Rio de Janeiro, 2013. 171p.

INSTITUTO DE PESQUISAS TECNOLÓGICAS DO ESTADO DE SÃO PAULO. Diagnóstico da situação atual dos recursos hídricos e estabelecimento de diretrizes técnicas para a elaboração do Plano da Bacia Hidrográfica do Tietê/Jacaré. São Paulo, 2000.

LORION, C. M.; KENNEDY, B. P. Relationships between deforestation, riparian forest buffers and benthic macroinvertebrates in neotropical headwater streams. Freshwater Biology, v. 54, n. 1, p. 165-180, 2009. http://dx.doi.org/10.1111/j.13652427.2008.02092.x 
MCDONALD-MADDEN, E.; BAXTER, P. W.; FULLER, R. A.; MARTIN, T. G.; GAME, E. T.; MONTAMBAULT, J. et al. Monitoring does not always count. Trends in

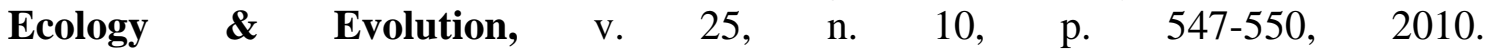
http://dx.doi.org/10.1016/j.tree.2010.07.002

MERRITT, D. M.; SCOTT, M. L.; LEROY, P. O. F. F.; AUBLE, G. T.; LYTLE, D. A. Theory, methods and tools for determining environmental flows for riparian vegetation: riparian vegetation-flow response guilds. Freshwater Biology, v. 55, n. 1, p. 206-225, 2010. http://dx.doi.org/10.1111/j.1365-2427.2009.02206.x

MICHALSKI, F.; METZGER, J. P.; PERES, C. A. Rural property size drives patterns of upland and riparian forest retention in a tropical deforestation frontier. Global Environmental Change, v. 20. n. 4, p. 705-712, 2010.

http://dx.doi.org/10.1016/j.gloenvcha.2010.04.010

MISERENDINO, M. L.; CASAUX, R.; ARCHANGELSKY, M.; DI PRINZIO, C. Y.; BRAND, C.; KUTSCHKER, A. M. Assessing land-use effects on water quality, instream habitat, riparian ecosystems and biodiversity in Patagonian northwest streams. Science of the Total Environment, v. 409, n. 3, p. 612-624, 2011. http://dx.doi.org/10.1016/j.scitotenv.2010.10.034

NEILL, C.; DEEGAN, L. A.; THOMAS, S. M.; CERRI, C. C. Deforestation for pasture alters nitrogen and phosphorus in small Amazonian streams. Ecological Applications, v. 11, n. $6, \quad$ p.1817-1828, 2001. http://dx.doi.org/10.1890/10510761(2001)011[1817:DFPANA]2.0.CO;2

OMETO, J. P. H.; MARTINELLI, L. A.; BALLESTER, M. V.; GESSNER, A.; KRUSCHE, A. V.; VICTORIA, R. L. et al. Effects of land use on water chemistry and macroinvertebrates in two streams of the Piracicaba river basin, south-east Brazil. Freshwater Biology, v. 44, n. 2, p. 327-337, 2000. http://dx.doi.org/10.1046/j.13652427.2000.00557.x

PETERSEN, R. C. The RCE: a riparian, channel, and environmental inventory for small streams in the agricultural landscape. Freshwater Biology, v. 27, n. 2, p. 295-306, 1992. http://dx.doi.org/10.1111/j.1365-2427.1992.tb00541.x

RITTER, W. F.; SHIRMOHAMMADI, A. (Eds.) Agricultural nonpoint source pollution: watershed management and hydrology. Boca Raton: CRC Press, 2010. 342p.

ROSENBERG, D. M.; RESH, V. H. Freshwater biomonitoring and benthic macroinvertebrates. New York: Chapman \& Hall, 1993. 488p.

SHELDON, F.; PETERSON, E. E.; BOONE, E. L.; SIPPEL, S.; BUNN, S. E.; HARCH, B. D. Identifying the spatial scale of land use that most strongly influences overall river ecosystem health score. Ecological Applications, v. 22, n. 8, p. 2188-2203, 2012. http://dx.doi.org/10.1890/11-1792.1

SOKAL, R. R.; ROHLF, F. J. Biometry: the principles and practice of statistics in biological research. 3. ed. New York: W. H. Freeman, 1995. 880p.

SOUZA, A. L. T.; FONSECA, D. G.; LIBÓRIO, R. A.; TANAKA, M. O. Influence of riparian vegetation and forest structure on the water quality of rural low-order streams in SE Brazil. Forest Ecology and Management, v. 298, p. 12-18, 2013. http://dx.doi.org/10.1016/j.foreco.2013.02.022 
SURIANO, M. T.; FONSECA-GESSNER, A. A.; ROQUE, F. O.; FROEHLICH, C. G. Choice of macroinvertebrate metrics to evaluate stream conditions in Atlantic Forest, Brazil. Environmental Monitoring and Assessment, v. 175, n. 1-4, p. 87-101, 2011. http://dx.doi.org/10.1007/s10661-010-1495-3

TUNDISI, J. G.; MATSUMURA-TUNDISI, T.; PARESCHI, D. C.; LUZIA, A. P.; VON HAELING, P. H.; FROLLINI, E. H. A bacia hidrográfica do Tietê/Jacaré: estudo de caso em pesquisa e gerenciamento. Estudos Avançados, v. 22, n. 63, p. 159-172, 2008. http://dx.doi.org/10.1590/S0103-40142008000200010

WYMAN, M. S.; STEIN, T. V. Modeling social and land-use/land-cover change data to assess drivers of smallholder deforestation in Belize. Applied Geography, v. 30, n. 3, p. 329-342, 2010. http://dx.doi.org/10.1016/j.apgeog.2009.10.001 